\title{
Services ecosystémiques du parc agroforestier à Cordyla pinnata (Lepr. ex A. Rich.) Milne-Redh. dans le Sud du Bassin Arachidier (Sénégal)
}

\author{
André Amakobo DIATTA ${ }^{1 *}$, Ngor NDOUR ${ }^{1}$, Alla MANGA ${ }^{2}$, Bienvenu SAMBOU $^{3}$, \\ Cheikh Sadibou FAYE ${ }^{3}$, Lamine Diatta ${ }^{3}$, Assane GOUDIABY ${ }^{3}$, Cheikh MBOW $^{3}$ et \\ Sara Danielle DIENG ${ }^{3}$
}
${ }^{1}$ Département d'Agroforesterie, Unité de Formation et de Recherche en Sciences et Technologie, Université Assane Seck de Ziguinchor, BP 523 Ziguinchor, Sénégal.
${ }^{2}$ Laboratoire d'Enseignement et de Recherche en Géomatique, Institut fondamental d'Afrique noire CAD, BP 206 Dakar, Université Cheikh Anta Diop de Dakar, Sénégal.
${ }^{3}$ Institut des Sciences de l'Environnement, Faculté des Sciences et Techniques, Université Cheikh Anta Diop de Dakar, BP 5005 UCAD Dakar, Sénégal.
*Auteur correspondant ; E-mail : andre_adiatta@yahoo.fr; Tel : 13148070734.

\section{RESUME}

Les parcs agroforestiers fournissent d'importants et multiples services aux populations vivant au Sud du Bassin Arachidier du Sénégal. Cependant, ces parcs sont confrontés à plusieurs facteurs de dégradation. Cette recherche, menée dans l'ancienne communauté rurale de Keur Samba Guèye (région de Fatick), vise à documenter les services écosystémiques fournis par le parc à Cordyla pinnata. L'approche méthodologique est basée sur des enquêtes socioéconomiques et ethnobotaniques auprès des populations locales et un inventaire des individus de $C$. pinnata et des espèces ligneuses associées. Les résultats de l'enquête révèlent que le parc contribue à l'amélioration de la fertilité des sols et fournissent des produits utilisés dans l'alimentation humaine et du bétail, la pharmacopée, le bois de service et d'énergie, et comme sources de revenus. Dans le parc agroforestier, le stock moyen de carbone des individus de $C$. pinnata est évalué à $8.81 \mathrm{tCO}_{2} \cdot \mathrm{ha}^{-1}$. L'ensemble de ces résultats devraient aider à sensibiliser les populations et à promouvoir une gestion rationnelle du parc agroforestier à $C$. pinnata.

(C) 2016 International Formulae Group. All rights reserved.

Mots clés : Agroforesterie, Cordyla pinnata, services écosystémiques, séquestration du carbone, Sénégal.

\section{Ecosystem services of Cordyla pinnata (Lepr. ex A. Rich.) Milne-Redh. agroforestry parkland of Senegal's South Peanut Basin}

\begin{abstract}
Agroforestry parklands provide multiple valuable services to farmers in the Southern Peanut Basin of Senegal. In order to understand ecosystem services provided by Cordyla pinnata agroforestry parkland, we conducted a study in the rural community of Keur Samba Gueye (Fatick, Senegal). The methodological approach was based on socioeconomic and ethnobotanical surveys with local populations and an inventory of C. pinnata trees and associated ligneous species. The results of the survey revealed that the agroforestry
\end{abstract}


parkland improves soil fertility and provides several products used in human consumption, livestock fodder, traditional medicine, construction and fuel, and income generation. The average carbon stock of $C$. pinnata trees in the agroforestry parkland is also estimated at $8.81 \mathrm{tCO}_{2} \cdot \mathrm{ha}^{-1}$. The results from this study could be used to raise awareness of the ecosystem services provided by $C$. pinnata agroforestry parkland to promote its sustainable management.

(C) 2016 International Formulae Group. All rights reserved.

Keywords: Agroforestry, Cordyla pinnata, ecosystem services, carbon sequestration, Senegal.

\section{INTRODUCTION}

Dans les régions semi-arides de l'Afrique de l'Ouest, particulièrement au Sud du Bassin Arachidier sénégalais, les parcs agroforestiers jouent un rôle fondamental pour les populations rurales (Diatta et al., 2016). Ils procurent aussi des produits utilisés dans l'alimentation humaine et du bétail, la pharmacopée, le bois de service et d'énergie (Sène, 2004 ; Yameogo et al., 2013; Laouali et al., 2014) et procurent des sources de revenus (Boffa, 2000) aux populations locales. D'un point de vue écologique, ils contribuent à l'atténuation des changements climatiques par leur capacité de séquestration du carbone (Mbow, 2009 ; Kumar et Nair, 2011) et jouent un rôle important dans la protection (Samba et al., 2012) et l'amélioration de la fertilité des sols (Samba, 2001; Saidou et al., 2012). Cependant, les systèmes de production agricole dans le Bassin Arachidier du Sénégal, en particulier le parc agroforestier à Cordyla pinnata (l'espèce est nommée «Dimb» en langue vernaculaire wolof; Bergeret, 1993), sont marqués par une dégradation progressive au fil des années (Herrmann et Tappan, 2013). Les principales contraintes du parc sont les coupes pour le bois d'énergie et le bois d'œuvre, les divers prélèvements (écorce, racine, feuilles...) de plantes médicinales, les feux de brousse et l'avancée du front pionnier agricole (Lykke, 2000). A cela s'ajoute la faible régénération des espèces et la lenteur de la croissance des principales espèces agroforestières à haute valeur ajoutée (Diop et al., 2011).
Aujourd'hui, l'état de dégradation du parc agroforestier à Cordyla pinnata ne semble pas le permettre de fournir tous les services associés à ce système d'utilisation et de gestion des arbres. Il apparait donc important de conduire la présente étude pour évaluer les services écosystémiques fournis par ces systèmes de production en rapport avec les besoins des communautés villageoises. Les résultats de cette étude devraient contribuer à la formulation de recommandations en termes de stratégies paysannes pour l'aménagement et la gestion durable des ressources naturelles des terroirs villageois.

\section{MATERIEL ET METHODES Zone d'étude}

Cette étude a été menée dans la commune «rurale» de Keur Samba Guèye (Suite à la Loi n 2013-10 du 28 décembre 2013 portant Code général des Collectivités locales, les communautés rurales sont devenues des communes; Figure 1) dans le Sud du Bassin Arachidier sénégalais au niveau des terroirs de Keur Samba Guèye $\left(13^{\circ} 39^{\prime} \mathrm{N}\right.$ et $\left.16^{\circ} 21^{\prime} \mathrm{O}\right)$ et de Santhie Rame $\left(13^{\circ} 38^{\prime} \mathrm{N}\right.$ et $\left.16^{\circ} 13^{\prime} \mathrm{O}\right)$. L'agriculture associée à un élevage à vocation agricole (parcage des animaux), un élevage allié à une agriculture de subsistance, la foresterie et les activités d'appoint telles que le petit commerce, le maraîchage et l'arboriculture, représentent les principales activités socioéconomiques des populations locales (PAGT, 2001).

La commune est caractérisée par un climat de type soudanien avec une longue 
saison sèche (novembre à mai) de sept mois et une saison pluvieuse qui dure cinq mois (juin à octobre). La pluviométrie moyenne annuelle de la commune est de $800 \mathrm{~mm}$ (PLD, 2012). Les trois principaux types de sols, au nombre de trois, sont les sols ferrugineux tropicaux lessivés ou Dior avec des caractéristiques sableuses $(70 \%)$, les sols hydromorphes ou Deck, argileux (10\%) et les sols sabloargileux ou Deck-Dior (20\%). La végétation recensée dans les sites étudiés (Figure 1) de la commune de Keur Samba Guèye est composée de 35 espèces ligneuses réparties en 34 genres et 18 familles dans les parcs agroforestiers à Cordyla pinnata (Diatta et al., 2016). Les travaux d'inventaire de Diatta et al. (2016) attestent que les espèces les plus représentées dans le parc sont Cordyla pinnata, Parkia biglobosa, Guiera senegalensis et Combretum glutinosum.

Le choix des sites (02) est basé sur des critères géographiques, historiques et sociaux et le niveau de dégradation du parc agroforestier avec une régression plus accentuée de la densité des arbres à Keur Samba Guèye comparée au site de Santhie Rame.

\section{Collecte des données socioéconomiques et ethnobotaniques.}

La collecte des données socioéconomiques et ethnobotaniques a été réalisée à travers une enquête auprès des populations locales pour connaître les biens et services qu'elles tirent du parc agroforestier à Cordyla pinnata. Cette enquête est faite par le biais d'entretiens structurés à l'aide de questionnaires auprès des ménages de chaque site avec un taux de sondage de $30 \%$ avec comme paramètres retenus l'activité socioprofessionnelle ensuite le genre. Les principaux aspects abordés dans le questionnaire sont: l'identification de l'enquêté, les services écosystémiques du parc agroforestier à $C$. pinnata, les usages de $C$. pinnata et les tendances évolutives de la ressource ligneuse du parc.

Des entretiens semi-structurés (individuels et de groupe) avec des personnes- ressources de la communauté ont aussi été administrés sur la base de guides d'entretien pour une meilleure connaissance des formes d'utilisation des espèces ligneuses du parc par les populations (Guèye et Freudenberger, 1991; Goudiaby, 1996). Les guides d'entretien ont pris en compte les caractéristiques des systèmes de productions, les différents domaines d'utilisation des espèces ligneuses $\mathrm{du}$ parc agroforestier à $C$. pinnata et la dynamique et les tendances évolutives du peuplement.

Méthode d'analyse des données
socioéconomiques et ethnobotaniques
L'exploitation des données socioéconomiques a été faite à l'aide du logiciel Sphinx Plus2. Les préférences des espèces ligneuses ont été définies à partir de la fréquence de citations ou d'indications des réponses obtenues auprès des populations locales. Les intérêts socioéconomiques de chaque espèce ligneuse (alimentation, pharmacopée, fourrage, énergie, construction, artisanat...) ont permis de connaître les services écosystémiques des parcs agroforestiers des sites sélectionnés.

\section{Méthode d'estimation du stock de carbone aérien et racinaire}

La méthodologie d'estimation du carbone séquestré par les individus de Cordyla pinnata est basée sur l'évaluation de la biomasse présente dans leurs composantes aériennes et souterraines. L'estimation de la biomasse est obtenue à l'aide de la méthode dite indirecte qui consiste, entre autres, à appliquer des modèles de régression allométrique sur des mesures dendrométriques effectuées sur le terrain (Valentini, 2007). La quantité de carbone séquestré est obtenue en multipliant la biomasse par le facteur d'expansion et de conversion de la biomasse par défaut qui équivaut à 0,5 .

$B A(k g)=1,929 \times d b h+0,116 \times d b h^{2}+0,013 \times d b h^{3}$

Mbow et al. (2014)

BRS $(\mathrm{kg})=\exp [-1,0587+0,8836 * \ln (\mathrm{BAS})]$

(Cairns et al., 1997). 
Où $\mathrm{BA}=$ Biomasse Aérienne ; $\mathrm{BAS}=$ Biomasse Aérienne Sèche, et BRS= Biomasse Racinaire Sèche

BAS $-\mathrm{CO}_{2}(\mathrm{~kg})=$ Carbone $(\mathrm{BAS}) * \mathbf{3 , 6 7}$

BRS - $\mathrm{CO}_{2}(\mathrm{~kg})=$ Carbone $(\mathrm{BRS}) * 3,67$

La méthode d'estimation du carbone racinaire est basée sur le ratio entre la biomasse souterraine et la biomasse sur pied. Le tableur Excel a permis de calculer le stock de carbone en multipliant le contenu en carbone de la biomasse aérienne et racinaire par 3,67 et de représenter sur des graphiques la proportion de carbone stocké dans le parc agroforestier à $C$. pinnata de la commune de Keur Samba Guèye.

\section{RESULTATS}

\section{Services écosystémiques du parc agroforestier à Cordyla pinnata Alimentation humaine}

Les résultats de l'enquête révèlent que les fruits verts de Cordyla pinnata entrent dans la composition des repas en guise de légumes avec $51 \%$ des produits destinés à l'autoconsommation et $35 \%$ à la vente. $C$. pinnata est ainsi utilisé par $14 \%$ des populations enquêtées pour son rôle primordial dans l'alimentation, en participant notamment à l'équilibre nutritionnel du menu quotidien (Figure 2). Il est l'espèce préférée à cet effet sur les sites pour $21 \%$ des populations locales (Figure 3). Les résultats de l'enquête indiquent que les principales espèces ligneuses du parc citées pour leur fonction alimentaire dans la commune de Keur Samba Guèye sont: Parkia biglobosa (15\%), Anacardium occidentale (13\%), Mangifera indica (10\%), Zyziphus mauritiana (10\%), Detarium senegalensis (8\%), Parinari macrophylla et Cola cordifolia (5\% chacune), et Adansonia digitata (4\%).

\section{Alimentation du bétail}

Dans la commune de Keur Samba Guèye, $89 \%$ des personnes interrogées estiment que l'élevage a un caractère extensifsédentaire. Les feuilles de Cordyla pinnata, souvent récoltées par élagage (47\%) ou émondage $(22 \%)$, constituent un fourrage apprécié par le bétail vers la fin de la saison sèche (mois d'avril et mai) selon $8 \%$ des populations enquêtées (Figure 2). Cependant, seulement $9 \%$ des populations locales indiquent qu'il est le principal ligneux fourrager du parc (Figure 3). Les principaux arbres utilisés dans l'alimentation du bétail par les populations enquêtées sont Pterocarpus erinaceus (17\%), Mangifera indica (14\%), Ficus capensis (7\%), Anacardium occidentale et Ficus sicomorus (6\% chacun), et Parkia biglobosa (4\%).

\section{Pharmacopée}

Les parcs agroforestiers sont des éléments essentiels des systèmes médicaux traditionnels. Pour Cordyla pinnata, $14 \%$ des populations estiment qu'il est utilisé dans le traitement des maux de ventre et des infections parasitaires (Figure 2) alors que $17 \%$ affirment qu'il est le ligneux le plus important dans le parc agroforestier en termes d'usage en pharmacopée (Figure 3). Les espèces ligneuses les plus utilisées dans la pharmacopée dans la commune de Keur Samba Guèye sont Pterocarpus erinaceus $(13 \%), \quad$ Guiera senegalensis $\quad(10 \%)$, Combretum glutinosum (6\%), Vitex madiensis et Parkia biglobosa (chacune 5\%), Ficus capensis et Terminalia macroptera (avec chacune $4 \%$ ).

\section{Bois de service et d'énergie}

Le bois de Cordyla pinnata représente la principale source de bois pour la confection d'outils et de matériaux divers dont certains ustensiles de cuisine, des perches, des piquets, des pirogues, des meubles pour $27 \%$ des populations interrogées, et comme source d'énergie domestique pour $19 \%$ des enquêtés (Figure 3). Il fait aussi l'objet d'une utilisation fréquente comme bois de service pour $27 \%$ des populations locales alors que $13 \%$ des populations enquêtées révèlent qu'il est utilisé comme bois de chauffage (Figure 2). Les résultats de l'enquête montrent que les espèces ligneuses préférées comme bois de service par les populations de la commune de 
Keur Samba Guèye sont Pterocarpus erinaceus (18\%), Khaya senegalensis $(12 \%)$, Azadirachta indica (7\%), Terminalia macroptera (7\%), Prosopis africana (6\%), Parkia biglobosa et Combretum glutinosum (avec chacune 4\%) de citations des enquêtés. Pour le bois de service obtenu des parcs agroforestiers, il est destiné à l'autoconsommation pour $71 \%$ des populations locales et $24 \%$ estiment qu'il est destiné à la vente.

Pour ce qui concerne le bois d'énergie dans la commune de Keur Samba Guèye, les principales espèces ligneuses qui font l'objet d'exploitation par les populations sont: Combretum glutinosum (14\%), Pterocarpus erinaceus (10\%), Parkia biglobosa et Terminalia macroptera $(9 \%$ chacune), Anacardium occidentale (8\%), Khaya senegalensis (6\%), Ficus capensis (5\%), et Guiera senegalensis (3\%). Les modes d'obtention du bois de chauffage dans la zone d'étude sont principalement le ramassage (51\%) et la coupe $(45 \%)$. Une grande partie du bois récolté est destinée à une utilisation familiale pour $77 \%$ des personnes enquêtées alors que $19 \%$ du bois récolté est vendu pour diversifier les sources de revenus selon les populations.

\section{Sources de revenus}

Les systèmes de production sont caractérisés par une agriculture de subsistance et un élevage à vocation agricole. Cordyla pinnata est l'espèce la plus appréciée par les populations locales pour les revenus qu'elle procure par la vente de ses sous-produits (bois, fruits, etc.) en plus de ses multiples services (Figure 2). En effet, les biens que les populations de la commune de Keur Samba Guèye tirent du parc sont importants et divers. Il s'agit de noix, de fruits, d'huiles, de condiments, de fourrages, de produits médicinaux, de fibres et de tanins.

\section{Protection des sols et amélioration de leur fertilité}

Les parcs agroforestiers sont des systèmes d'utilisation des terres qui contribuent à la protection des sols et à l'amélioration de leur fertilité d'autant plus que $96 \%$ des populations enquêtées estiment que la fertilité des terres a diminué avec des rendements faibles à moyens pour $77 \%$ des participants. Pour $23 \%$ des enquêtés, Cordyla pinnata est conservé dans le parc pour sa capacité à protéger et à améliorer le sol (Figure 2) et $34 \%$ le préfèrent aux autres espèces ligneuses du parc agroforestier (Figure 3). En plus de $C$. pinnata, les principales espèces utilisées dans la protection des sols ou l'amélioration de leur fertilité par ordre d'importance sont: Parkia biglobosa (14\%), Faidherbia albida (10\%), Anacardium occidentale (8\%), Ficus capensis (6\%), Pterocarpus erinaceus (5\%), Guiera senegalensis, Combretum glutinosum et Terminalia macroptera avec chacune $3 \% \mathrm{du}$ pourcentage des réponses fournies par les enquêtés. En effet, la perte des feuilles des arbres au début de la saison des pluies et les excréments des animaux qui parcourent les parcs participent à l'amélioration de la fertilité des sols (Figure 4).

\section{Stock de carbone du parc agroforestier à Cordyla pinnata}

Le stock de carbone des individus de C. pinnata du parc agroforestier est défini comme la quantité de carbone stocké dans la biomasse vivante au-dessus du sol et de la biomasse souterraine. La Figure 5 montre le stock de carbone aérien et racinaire des individus de $C$. pinnata de la commune de Keur Samba Guèye.

Le stock de carbone aérien des individus de $C$. pinnata du parc agroforestier est évalué à $683,87 \mathrm{tCO}_{2}$ ce qui correspond à $7,74 \mathrm{tCO}_{2} \cdot \mathrm{ha}^{-1}$. Quant au stock de carbone racinaire des individus de $C$. pinnata, il est égal à $94,19 \mathrm{tCO}_{2}$ soit $1,07 \quad \mathrm{tCO}_{2} \cdot \mathrm{ha}^{-1}$ et représentent $14 \%$ des stocks aériens. Ainsi, le carbone total stocké par les individus de $C$. pinnata du parc agroforestier est égal à 778,06 $\mathrm{tCO}_{2}$ ce qui correspond à $8.81 \mathrm{tCO}_{2} \cdot \mathrm{ha}^{-1}$.

Dans la commune de Keur Samba Guèye, l'essentiel du carbone est stocké dans les classes supérieures à $50 \mathrm{~cm}$ de diamètre et représente $91 \%$ du total des individus recensés. Les individus des classes 
[40-50 $\mathrm{cm}$ [ de diamètre ne font que $8 \%$ des effectifs alors que les individus de diamètre inférieur à $40 \mathrm{~cm}$ ne représentent que $1 \%$ des individus inventoriés (Figure 6). Le peuplement de $C$. pinnata dans les parcs agroforestiers est caractérisé par une abondance d'individus dans les grandes classes de diamètre et une régénération naturelle relativement faible.

\section{Tendances évolutives de la végétation ligneuse}

La densité de la végétation ligneuse du parc agroforestier à Cordyla pinnata dans la commune de Keur Samba Guèye dans le Sud du Bassin Arachidier est qualifiée de faible par $41 \%$ de la population enquêtée. Pour $39 \%$ des populations locales, la densité de la végétation ligneuse est qualifiée de moyenne alors que $18 \%$ estime qu'elle est abondante dans le parc. Selon 96\% des populations enquêtées de Keur Samba Guèye le parc à $C$. pinnata a connu une diminution de son effectif entre 1970 et 2012. Les principaux facteurs à l'origine de cette diminution sont les coupes (45\%), les feux $(15 \%)$, la pression sur les terres agricoles $(10 \%)$, la baisse de la pluviosité $(6 \%)$, et les plantes parasites pour 6\% (Figure 7).

Concernant l'avenir du couvert végétal, $88,6 \%$ des populations locales pensent que les espèces auront une évolution décroissante. Pour atténuer les effets négatifs des principaux facteurs (Figure 8) sur la dynamique du parc agroforestier, certaines stratégies ont été préconisées par les populations locales telles que l'initiation de programmes de reboisement $(31 \%)$, la sensibilisation et la formation des populations $(28 \%)$ et la mise en place de pare-feu $(21 \%)$. Ces stratégies pourraient aider à la volonté de conservation et de réhabilitation du parc agroforestier d'autant plus que $96 \%$ de la population enquêtée estiment qu'une diminution des arbres dans le parc s'accompagnerait d'une réduction des biens et services.

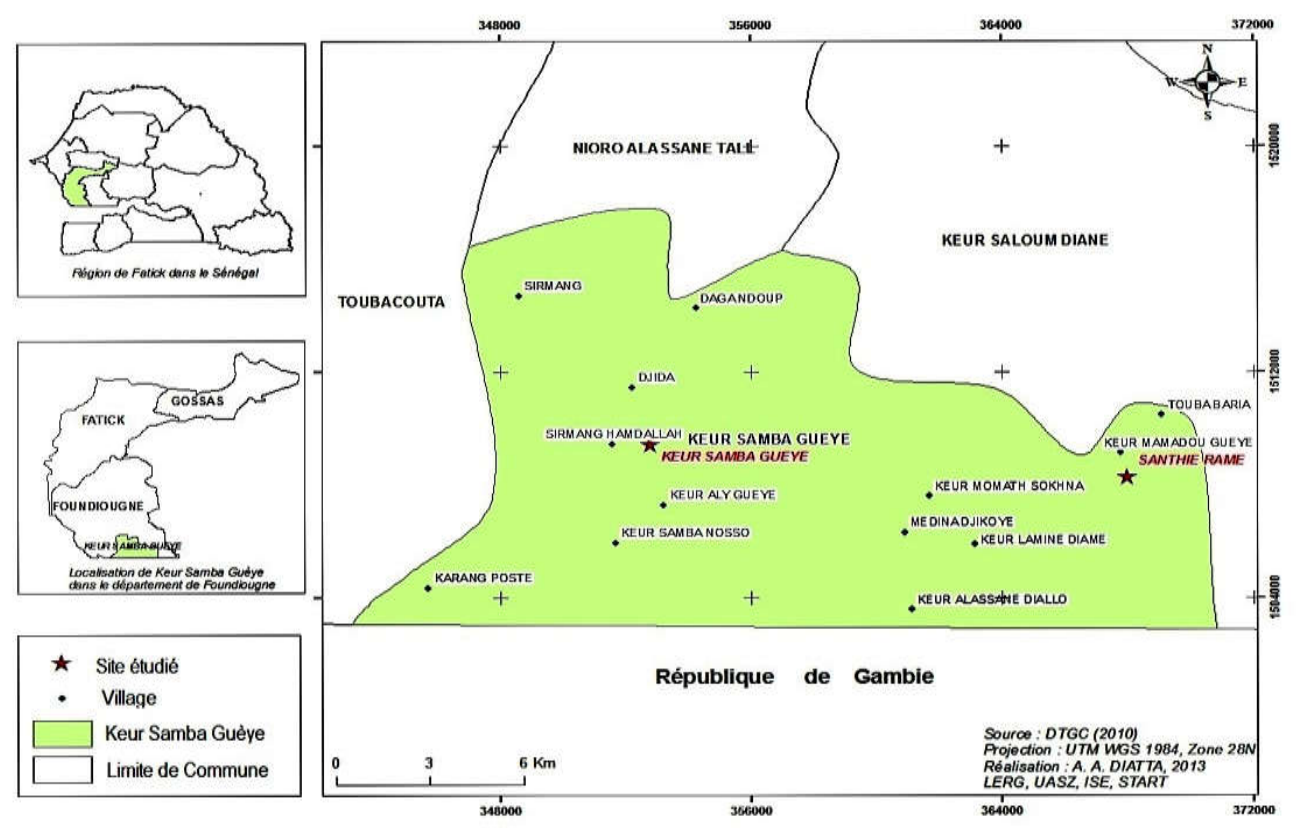

Figure 1: Carte de localisation des sites de l'étude. 


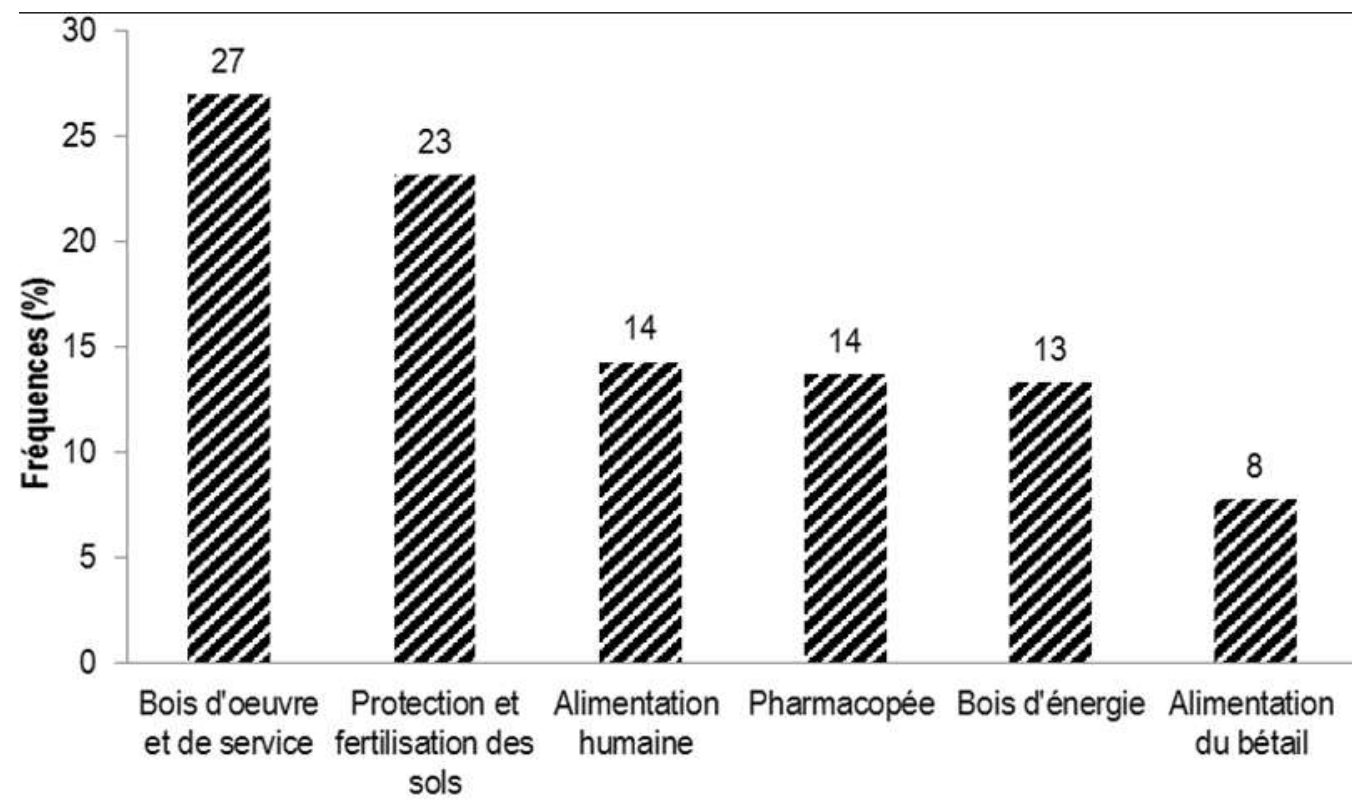

\section{Utilisations}

Figure 2 : Fréquences de citations des différents usages de Cordyla pinnata par les paysans.

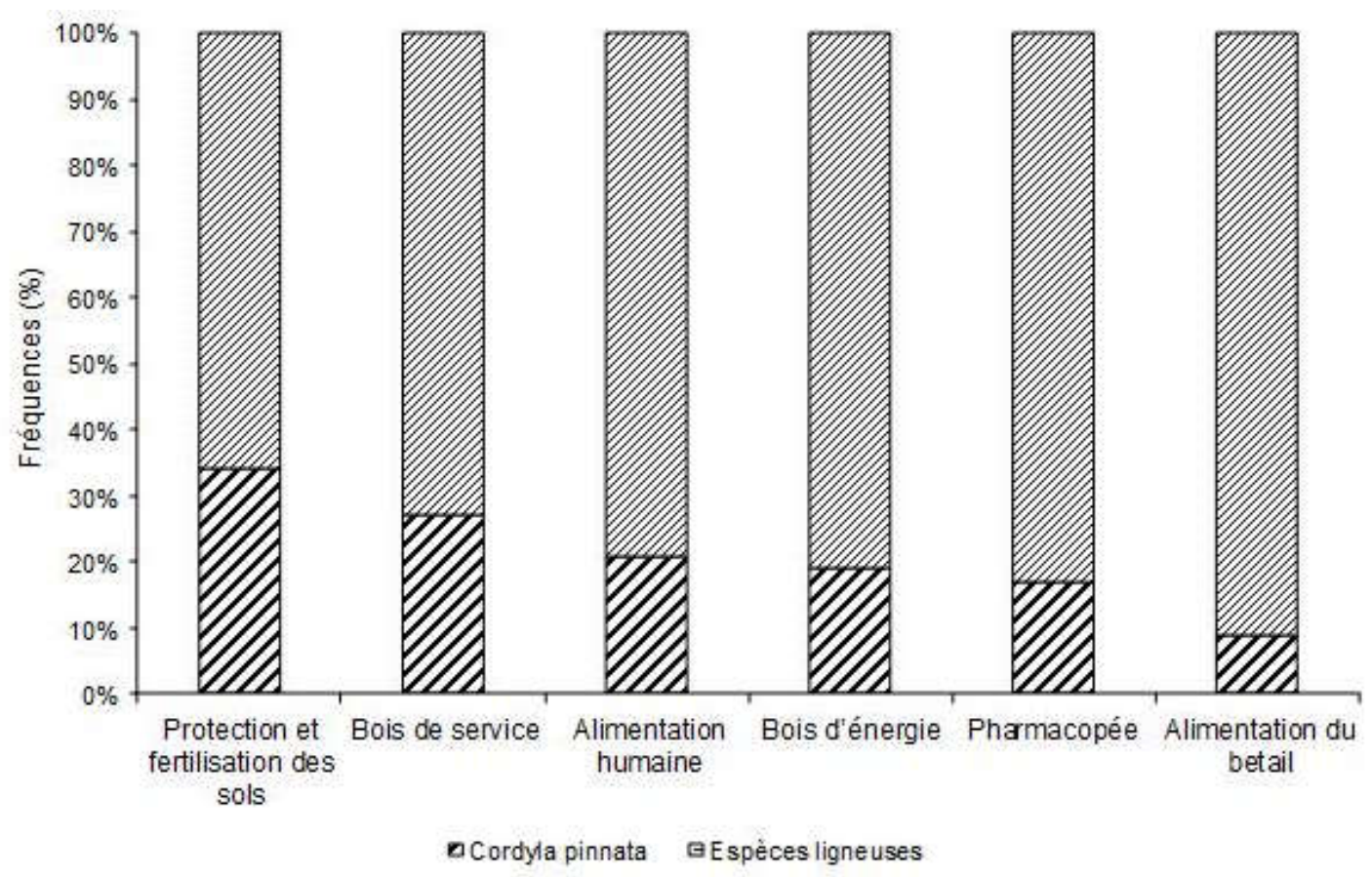

Figure 3 : Fréquences de la préférence de Cordyla pinnata comparées aux espèces ligneuses du parc agroforestier pour les différents usages. 


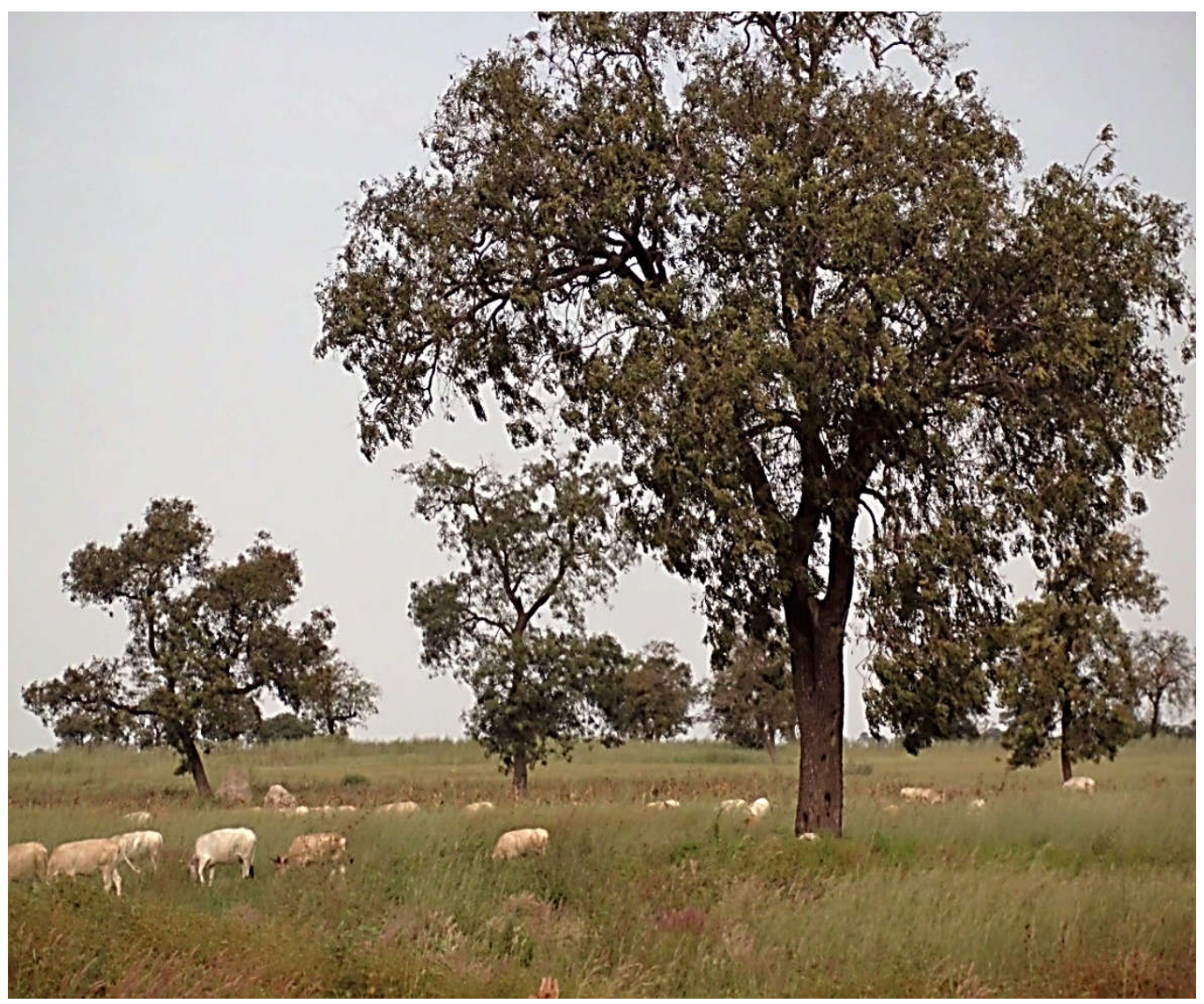

Figure 4 : Parc agroforestier à Cordyla pinnata .

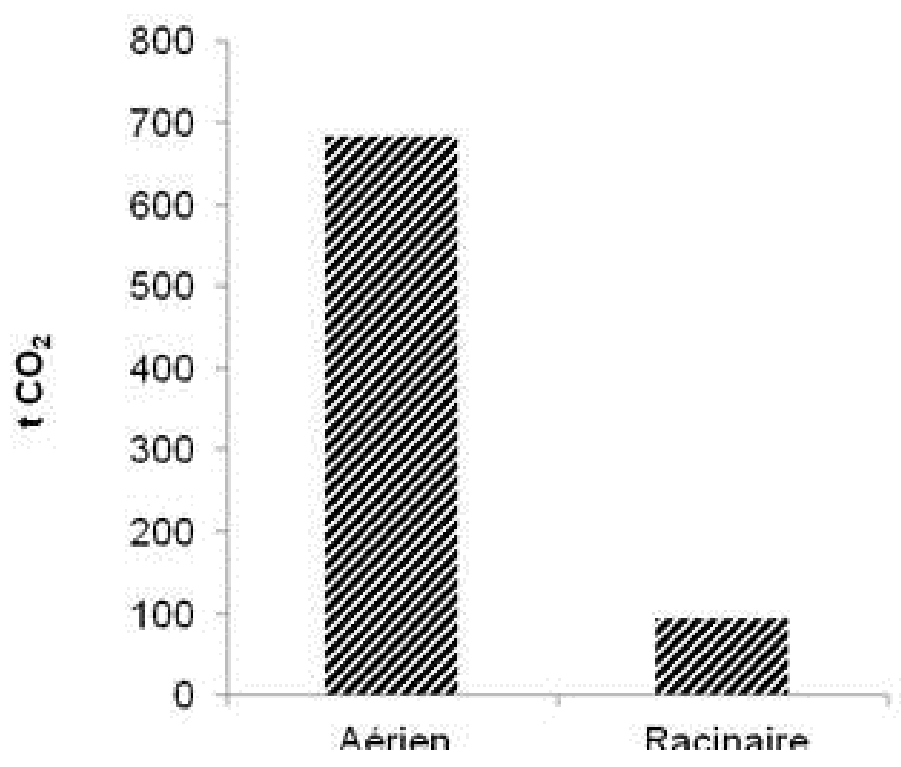

Figure 5 : Stock de carbone aérien et racinaire des individus de Cordyla pinnata. 


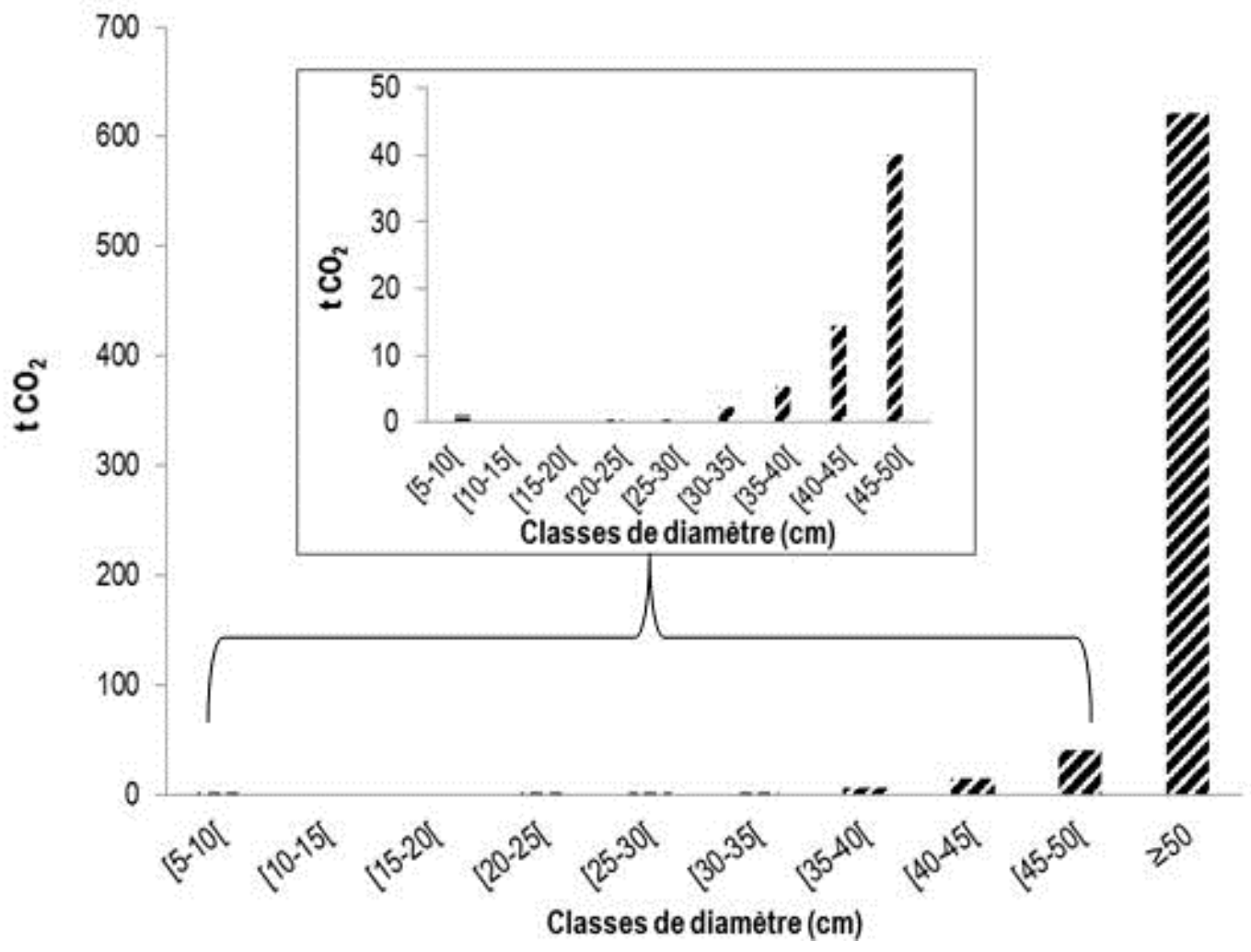

Figure 6 : Stock de carbone par classe de diamètre des individus de Cordyla pinnata.

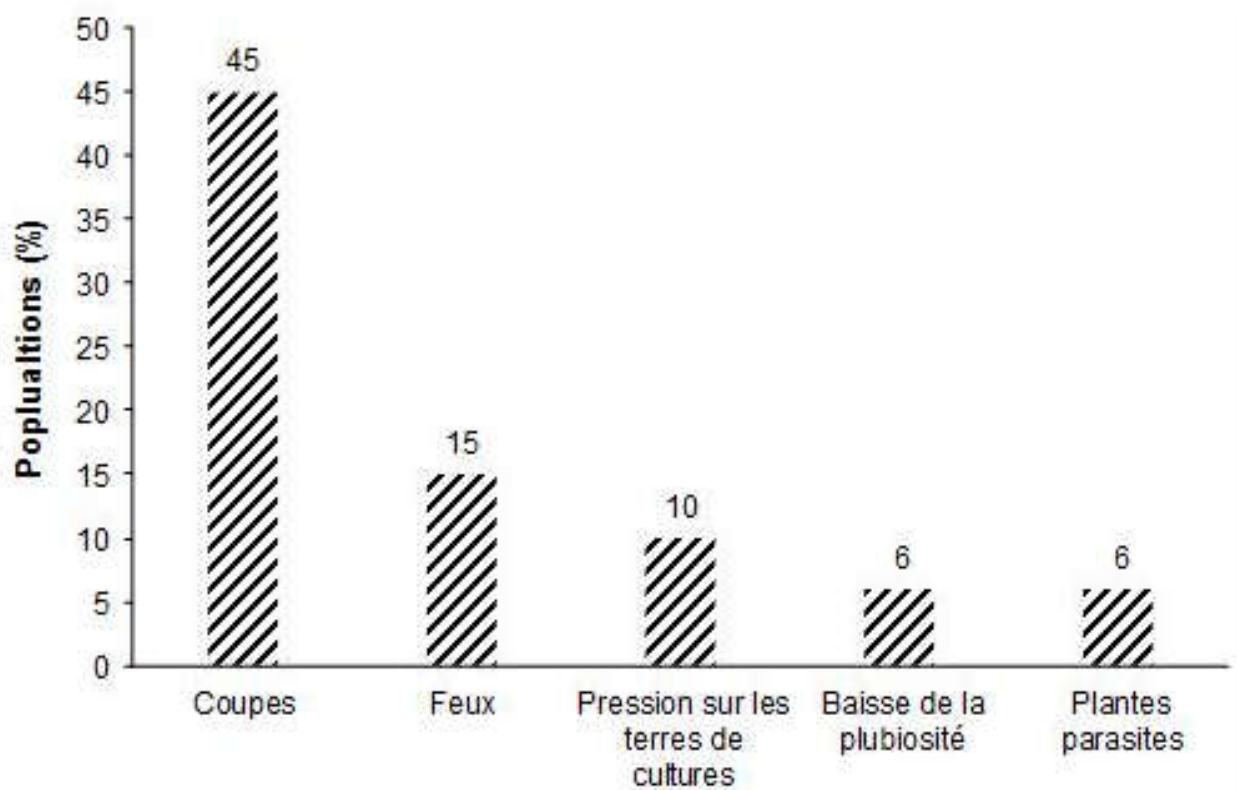

Figure 7 : Les principaux facteurs expliquant les tendances évolutives de la végétation ligneuse du parc agroforestier à Cordyla pinnata . 


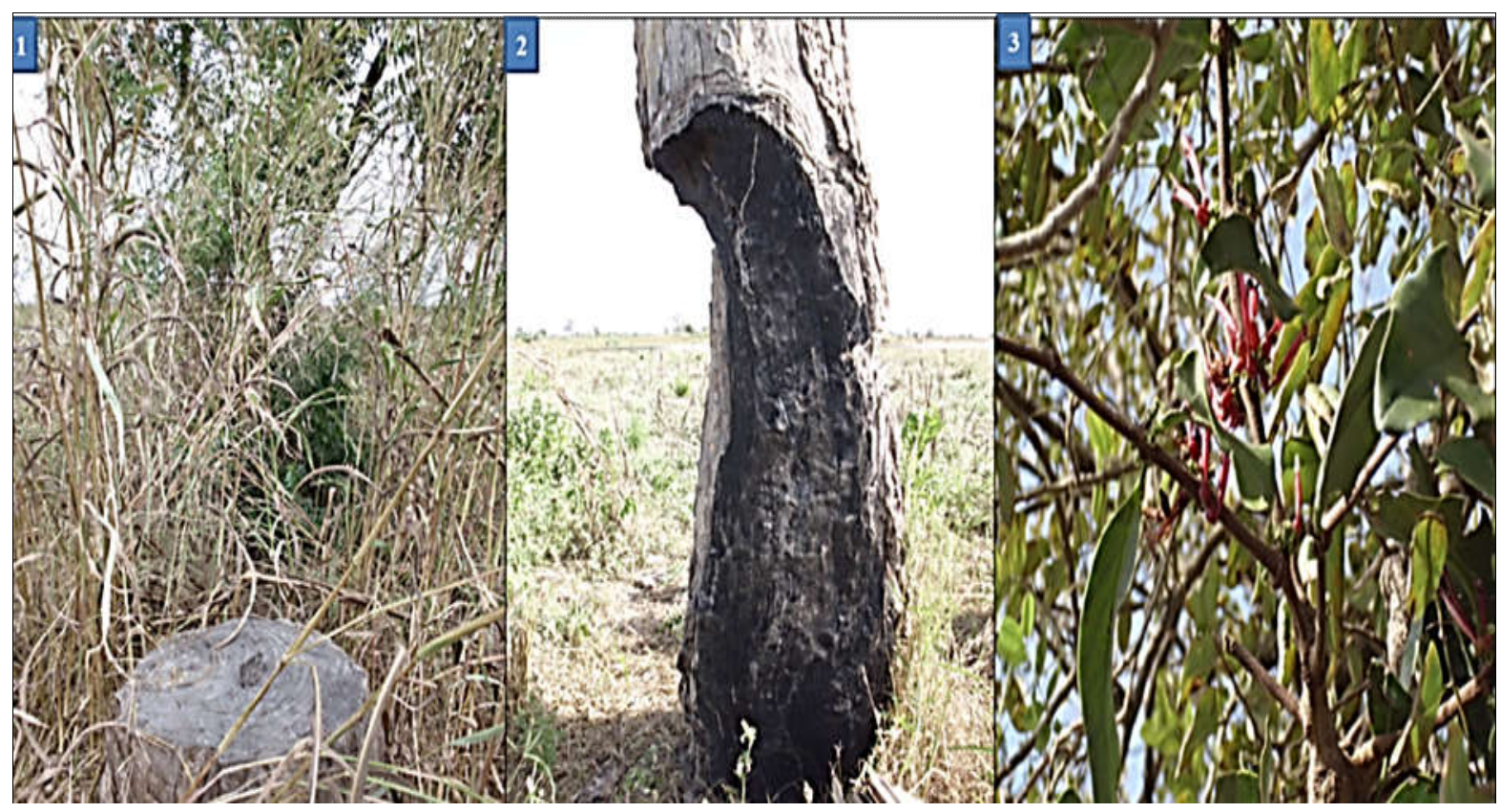

Figure 8 : Facteurs de dégradation des parcs : 1. Coupe ; 2. Impacts des feux ; 3. Plantes parasites (Tapinanthus sp.).

\section{DISCUSSION}

Le parc agroforestier à Cordyla pinnata fournit de multiples services écosystémiques aux populations locales. $C$. pinnata est particulièrement apprécié pour ses fruits riches en vitamine $C$, phosphore et fer, bien équilibrés en acides aminés essentiels et peuvent aussi compenser le déficit des céréales en lysine. Le parc agroforestier constitue une source importante dans l'alimentation humaine et du bétail, la pharmacopée, la protection des sols et l'amélioration de leur fertilité. Ces arguments corroborent les résultats de Diop et al. (2005) et Yameogo et al. (2013) qui attestent que les parcs jouent un rôle capital dans la vie des populations locales. Sur les sites d'étude, le parc fournit des produits alimentaires et médicinaux, du fourrage, du bois d'énergie et $\mathrm{du}$ bois nécessaire à la construction des habitats et à la fabrication d'outils et d'ustensiles de cuisine. Le parc agroforestier contribue à diversifier les produits alimentaires, à maintenir l'équilibre alimentaire à différentes périodes de l'année et à augmenter les revenus des populations locales (Boffa, 2000 ; Dieng et al., 2016). En effet, du fait de l'importance des parcs à $C$. pinnata dans l'alimentation dans le Sud du Bassin Arachidier et de sa qualité nutritive (Ayessou et al., 2011), l'espèce est considérée comme «arbre grenier» (Bérhaut, 1988) et son fruit appelé « viande du Saloum » par les populations (Niang, 1990).

Dans la commune de Keur Samba Guèye, le rôle des espèces ligneuses dans l'alimentation des animaux est considérable. Laouali et al. (2014) ont aussi relevé l'utilisation de Prosopis africana comme fourrage par les populations dans le Sud du département d'Aguié au Niger. Les espèces ligneuses $\mathrm{du}$ parc agroforestier sont aussi utilisées par les populations locales dans le traitement des maladies de l'homme et des animaux. En effet, les feuilles de $C$. pinnata sont employées en pharmacopée dans le 
traitement des maux de ventre et des conjonctivites (Niang, 1990). Les décoctions de racines et d'écorce et la sève sont respectivement utilisées contre les maladies vénériennes et comme un laxatif naturel dans la pharmacopée animale (Sène, 1994 ; Sall, 1996 ; Ngom et al., 2014).

Quant à la production de bois, les espèces ligneuses $\mathrm{du}$ parc agroforestier peuvent fournir une part appréciable du bois utilisé comme bois de chauffe et de service (Baumer, 1997; Sarr et al., 2013). Cet argument corrobore les résultats de Sall (1996) et Dieng et al. (2016) qui témoignent que le bois de $C$. pinnata est utilisé dans la construction de pirogue, la charpenterie, la fabrication de meubles, dans l'artisanat local et dans la production de charbon de bois.

Les arbres de parcs agroforestiers se caractérisent par leur enracinement plus profond par rapport aux arbres forestiers (Dupraz et Liagre, 2008), une faible concurrence entre individus et une fertilisation des cultures associées entrainant une augmentation de la biomasse des individus (Hamon et al., 2009). Le parc agroforestier peut jouer aussi un rôle important dans la protection des sols et l'amélioration de leur fertilité par la modification de leurs propriétés physico-chimiques (Diallo et al., 2006). Cette analyse soutient les observations de Boffa (2000) qui considère que l'un des principaux avantages de l'intégration des arbres dans un système agroforestier est l'amélioration de la fertilité des sols avec un accroissement de la fertilité sous le houppier des arbres. Cordyla pinnata a un grand potentiel de production de biomasse foliaire et devrait assurer au sol des apports non négligeables de matière organique sous forme de litière. Ces apports d'éléments nutritifs au sol améliorent les conditions micro environnementales des sites et augmentent généralement la productivité des cultures agricoles (Samba, 2001). Grouzis et Akpo
(2006) rapportent que l'arbre modifie les conditions physiques du sol et améliore les conditions trophiques à son voisinage aussi bien en zone de savanes que dans les parcs. On note également une augmentation de la biomasse herbacée sous le couvert des arbres du parc agroforestier (Bonkoungou et al., 1997).

Le parc agroforestier à Cordyla pinnata de la commune de Keur Samba Guèye constitue un important réservoir de carbone de par sa structure et les quantités de carbone stocké dans la biomasse des arbres. Cet argument confirme les résultats de Diatta (2013) qui attestent que la population de Faidherbia albida des parcs agroforestiers dans le Nord du Bassin Arachidier du Sénégal a un potentiel de 7,1 $\mathrm{tCO}_{2} \cdot \mathrm{ha}^{-1}$. Ces caractéristiques montrent que le parc agroforestier dans le Bassin Arachidier sénégalais dans un état de dégradation poussée entrainerait un déséquilibre dans les flux de carbone. Il serait donc, dans le long ou moyen terme, davantage un réservoir de carbone qu'un puits. Cependant, les travaux de Marone et al. (2016) rapportent un stock total de carbone de la biomasse des parcs agroforestiers évalué à $36 \mathrm{tCO}_{2} \cdot \mathrm{ha}^{-1}$ dans la zone semi-aride des Niayes du Sénégal. Ces parcs agroforestiers constitueraient donc des secteurs dynamiques et prometteurs de l'atténuation des changements climatiques (Mbow, 2010).

\section{Conclusion}

Ce travail a permis de mettre en exergue les multiples services écosystémiques fournis par le parc agroforestier à Cordyla pinnata aux populations des sites de Keur Samba Guèye et Santhie Rame au Sud du Bassin Arachidier. Le parc fournit des produits dans l'alimentation humaine et du bétail, dans la pharmacopée, et du bois de service et d'énergie et procure des sources de 
revenus aux populations locales. Il contribue à la protection des sols et à l'amélioration de leur fertilité et à la séquestration du carbone. Cependant, il apparaît une dégradation de sa structure associée à une faiblesse de la régénération des espèces ligneuses avec comme conséquence une diminution des services écosystémiques à cause de l'augmentation des besoins des populations en produits agroforestiers, un accroissement de la pauvreté en milieu rural et une baisse des rendements des systèmes de production. Sur la base de ces résultats, il serait donc intéressant de poursuivre la réflexion sur les possibilités et les logiques paysannes de conservation et de gestion durable des parcs agroforestiers au Sud du Bassin arachidier.

\section{CONFLIT D'INTERETS}

Les auteurs déclarent qu'il n'y a aucun conflit d'intérêt qui serait liée au présent article.

\section{CONTRIBUTIONS DES AUTEURS}

AAD: Elaboration de la méthodologie de recherche, inventaire des parcs agroforestiers, enquêtes socioéconomiques et ethnobotaniques, analyse des données et rédaction de l'article; $\mathrm{NN}$ : Co-encadreur du travail, négociateur du stage de André Amakobo Diatta à l'Institut des Sciences de l'Environnement (ISE) de l'Université Cheikh Anta Diop (UCAD) de Dakar, contribution à la rédaction de la méthodologie de recherche, à la correction du mémoire de Master de l'auteur principal, à l'analyse des données et la rédaction de l'article; BS: Directeur de Mémoire de André Amakobo Diatta, accueil $\mathrm{du}$ stagiaire, contribution au choix des sites d'étude, à la rédaction de la méthodologie de recherche et à la correction du mémoire d'où est tiré ce présent article; $\mathrm{AM}$ et $\mathrm{CM}$ : Coordonnateurs, responsables de la gestion administrative du projet «Application of Earth
Observation methods for monitoring and assessment of agro forest performances in Senegal», contribution au choix des images satellitaires et à la rédaction de l'article ; CSF, LD, AG et SDD: Collaborateurs dans la collecte et le traitement des données.

\section{REMERCIEMENTS}

Ce travail est l'aboutissement d'une collaboration entre le Département Agroforesterie de l'Université Assane Seck de Ziguinchor, de l'Institut des Sciences de l'Environnement et $\mathrm{du}$ Laboratoire d'Enseignement et de Recherche en Géomatique de l'Université Cheikh Anta Diop de Dakar, du Département de Botanique de l'Université de Ghana et du Département de Foresterie de l'Université de Michigan à travers le projet START (SysTem for Analysis, Research and Training) que nous remercions pour nous avoir donné les moyens financiers et logistiques nécessaires à la réalisation de ce travail. Nous remercions aussi les populations de Keur Samba Guèye et Santhie Rame pour leur participation à cette étude.

\section{REFERENCES}

Ayessou NC, Ndiaye C, Cissé M, Gueye M, Sakho M. 2011. Nutritional contribution of some Senegalese Forest fruits running across Soudano-Sahelian Zone. Food and Nutrition Sciences, 2(6): 606.

Baumer M. 1997. L'Agroforesterie pour les Productions Animales. ICRAF: Nairobi, Kenya ; 340 p.

Bergeret A. 1993. Le Dimb (Cordyla pinnata). Le Flamboyant, 27: 14-15.

Bérhaut J. 1988. Flore Illustrée du Sénégal (Tomes I-IX.). Gouvernement du Sénégal, Ministère du développement rural, Direction des eaux et forêts. Clairafrique : Dakar. 
Boffa JM. 2000. Les Parcs Agroforestiers en Afrique Subsaharienne. Cahier FAO Conservation 34: Rome.

Bonkoungou EG, Ayuk ET, Zoungrana I. 1997. Les parcs agroforestiers des zones semi-arides d'Afrique de l'Ouest. Actes $\mathrm{du}$ séminaire international, ICRAF/IRBET/CILSS/LTC, Ouagadougou, Burkina Faso, 25-27 Oct. 1993. Centre International pour la Recherche en Agroforesterie (ICRAF) : Nairobi.

Bonneville J, Demers N. 2005. Recherche en agroforesterie et développement au Sahel : De nouveaux objectifs pour poursuivre une collaboration fructueuse. Sahel Agroforesterie Sahel $n^{\circ} 4$.

Cairns MA, Brown S, Helmer EH, Baumgardner GA. 1997. Root biomass allocation in the world's upland forests. Oecologia, 111(1): 1-11.

Diatta AA, Ndour N, Manga A, Sambou B, Faye CS, Diatta L, Mbow C. Composition floristique et dynamique du parc agroforestier à Cordyla pinnata (Lepr. ex A. Rich.) Milne-Redh. dans le Sud du Bassin Arachidier (Sénégal). Int. J. Biol. Chem. Sci., 10(4): 1805-1822.

Diatta L. 2013. Contribution à la connaissance de l'état, de la dynamique et des services écosystémiques du parc agroforestier à Faidherbia albida (Del.) A. Chev. dans la communauté rurale de Réfane/Région de Diourbel, Sénégal. Mémoire de Master, Faculté des Sciences et Techniques, Institut des Sciences de l'Environnement, Université Cheikh Anta Diop de Dakar, Sénégal, p. 76.

Diallo MD, Duponnois R, Guisse A, Sall S, Chotte JL, Thioulouse J. 2006. Biological effects of native and exotic plant residues on plant growth, microbial biomass and $\mathrm{N}$ availability under controlled conditions. European Journal of Soil Biology, 42(4): 238-246.

Dieng SD, Diop M, Goudiaby A, Niang-Diop F, Faye LC, Guiro I, Sambou S, Lykke, AM, Sambou B. 2016. Caractérisation des services écosystémiques fournis par Cordyla pinnata dans la périphérie de la Forêt classée de Patako au Sénégal. VertigO-la Revue Electronique en Sciences de l'Environnement, 16(2).

Diop FN, Lykke AM, Sambou B. 2011. Régénération naturelle de Cordyla pinnata (Lepr. Ex. A. Rich.) MilneRedh. dans une savane soumise au feu et au pâturage. Science et changements planétaires/Sécheresse, 22(3): 186-191.

Diop M, Kaya B, Niang A, Olivier A. 2005. Les espèces ligneuses et leurs usages: les préférences des paysans dans le Cercle de Ségou au Mali. Nairobi: ICRAF Working Paper, 9, p.29.

Dupraz C, Liagre F. 2008. Agroforesterie, des Arbres et des Cultures. France Agricole Editions: Paris.

Goudiaby A. 1996. Etude de la flore et de la végétation de Dindefello (Sud-Est du Sénégal) : Eléments pour un aménagement. Thèse de Doctorat de $3^{\text {ème }}$ Cycle, Institut des Sciences de l'Environnement, Faculté des Sciences et Techniques, Université Cheikh Anta Diop de Dakar (UCAD), p. 81.

Grouzis M, Akpo LE. 2006. Interactions arbre-herbe au Sahel. Sécheresse, 17 (12): 318-25.

Guèye B, Freudenberger KS. 1991. Introduction à la Méthode Accélérée de Recherche Participative (MARP) Rapid Rural Appraisal. Quelques Notes pour Appuyer une Formation Pratique (2 $2^{\text {nde }}$ édition). IIED: Londres.

Hamon X, Dupraz C, Liagre F. 2009. L'Agroforesterie : Outil de séquestration du carbone en agriculture, $18 \mathrm{p}$. 
Herrmann SM, Tappan GG. 2013. Vegetation impoverishment despite greening: A case study from central Senegal. Journal of Arid Environments, 90: 55-66.

Kumar BM, Nair PKR. 2011. Carbon Sequestration Potential of Agroforestry Systems. Opportunities and Challenges Series (Vol. 8). Springer Science \& Business Media: New York.

Laouali A, Guimbo ID, Larwanou M, Inoussa MM, Mahamane A. 2014. Utilisation de Prosopis africana (G. et Perr.) Taub dans le sud du département d'Aguié au Niger: les différentes formes et leur importance. Int. J. Biol. Chem. Sci., 8(3), 1991-8631.

Lykke AM. 2000. Local perceptions of vegetation change and priorities for conservation of woody-savanna vegetation in Senegal. Journal of Environmental Management, 59(2): 107120.

Marone D, Poirier V, Coyea M, Olivier A, Munson AD. 2016. Carbon storage in agroforestry systems in the semi-arid zone of Niayes, Senegal. Agroforestry Systems: 1-14.

Mbow C. 2009. Potentiel et dynamique des stocks de carbone des savanes soudaniennes et soudano-guinéennes du Sénégal. Thèse de Doctorat d'Etat, Institut des Sciences de l'Environnement, Faculté des Sciences et Techniques, Université Cheikh Anta Diop de Dakar (UCAD), 319p.

Mbow C. 2010. Africa's risky gamble. Global Change Newsletter, 75: 20-23.

Mbow C, Verstraete MM, Sambou B, Diaw AT, Neufeldt H. 2014. Allometric maize) en zone Soudanienne du Bénin. Int. J. Biol. Chem. Sci., 6(5): 2066-2082.

Sall PN. 1996. Les parcs agroforestiers au Sénégal. Etat des connaissances et perspectives. Rapport AFRENA. Centre models for aboveground biomass in dry savanna trees of the Sudan and SudanGuinean ecosystems of Southern Senegal. Journal of Forest Research, 19: 340-347.

Niang MM. 1990. Contribution à la connaissance et à la valorisation des systèmes agroforestiers traditionnels au Sud du bassin Arachidier (Sénégal) : Cas du système à parc à Cordyla pinnata. Mémoire de diplôme d'Ingénieur des Eaux, Forêts et Chasses, CU de DSCHANG/DRPF-ISRA, p. 52.

Ngom D, Charahabil MM, Sarr O, Bakhoum A, Akpo LE. 2014. Perceptions communautaires sur les services écosystémiques d'approvisionnement fournis par le peuplement ligneux de la Réserve de Biosphère du Ferlo (Sénégal). VertigO-la Revue Electronique en Sciences de l'Environnement, 14(2).

PAGT. 2001. Plan d'Aménagement et de Gestion du Terroir de la Communauté Rurale de Keur Samba Guèye (Fatick, Sénégal). Rapport PAGERNA, MJEHP et GTZ. PRODDEL: Dakar, p. 50.

PLD. 2012. Plan Local de Développement de la Communauté Rurale de Keur Samba Guèye (Fatick, Sénégal). Rapport PBA/GTZ. PRODDEL : Dakar.

République du Sénégal. 2013. Loi n 2013-10 du 28 décembre 2013 portant Code général des Collectivités locales. $44 \mathrm{p}$.

Saidou A, Balogoun I, Kone B, Gnangle CP, Aho N. 2012. Effet d'un système agroforestier à karité (Vitellaria paradoxa cf gaertn) sur le sol et le potentiel de production du maïs (Zea international pour la recherche en agroforesterie (ICRAF) : Nairobi.

Samba ANS. 2001. Effet de la litière de Cordyla pinnata sur les cultures: approche expérimentale en 
agroforesterie. Ann. For. Sci., 58(1): 99107.

Samba SN, Faye E, Tala G, Hank M, Camire C. 2012. Cordyla pinnata améliore les propriétés du sol et la productivité des cultures. Int. J. Biol. Chem. Sci., 6(2): 714-725.

Sarr O, Bakhoum A, Diatta S, Akpo LE. 2013. L'arbre en milieu soudano-sahélien dans le bassin arachidier (Centre-Sénégal). Journal of Applied Biosciences, 61, 4515-4529. DOI 10.4314/jab.v61i0.85598

Sène A. 1994. Etude socio-économique des systèmes à parc dans le bassin arachidier: Cas de Sterculia setigera et de Cordyla pinnata. Mémoire de confirmation, Institut Sénégalais de Recherches Agricoles (ISRA), 64p.

Sène A. 2004. Dynamique et gestion paysanne des parcs agroforestiers dans le bassin arachidier du Sénégal. ICRAF (International Center for Research in Agroforestry), $18 \mathrm{p}$.

Valentini SG. 2007. Évaluation de la séquestration du carbone dans des plantations agroforestières et des jachères issues d'une agriculture migratoire dans les territoires autochtones de Talamanca, au Costa Rica. Mémoire pour l'obtention du grade de Maître ès Science (M.Sc.), Faculté des Sciences de L'agriculture et de l'alimentation Université Laval Québec, $89 \mathrm{p}$.

Yameogo G, Yelemou B, Boussim IJ, Traore D. 2013. Gestion du parc agroforestier du terroir de Vipalogo (Burkina Faso): contribution des ligneux à la satisfaction des besoins des populations. Int. J. Biol. Chem. Sci., 7(3): 1087-1105. 